\title{
On the evaluation of patient specific IMRT QA using EPID, dynalog files and patient anatomy
}

\author{
Dewayne Lee Defoor ${ }^{1,2}$, Panayiotis Mavroidis ${ }^{1,2}$, L Vazquez Quino ${ }^{1,2}$, Alonso Gutierrez ${ }^{1,2}$, \\ Niko Papanikolaou ${ }^{1,2}$, Sotiri Stathakis ${ }^{1,2}$ \\ ${ }^{I}$ Department of Radiation Oncology, University of Texas Health Science Center at San Antonio, San Antonio, TX, USA. \\ ${ }^{2}$ Cancer Therapy and Research Center, San Antonio, TX, USA.
}

Received March 19, 2014; Published Online April 08, 2014

\begin{abstract}
[Presented at the Young Investigator's Symposium at the 2014 Annual Meeting of Southwest Chapter of American Association of Physicists in Medicine (AAPM) in San Antonio, Texas, USA]
\end{abstract}

\section{Conference Proceeding}

\begin{abstract}
Purpose: This research, investigates the viability of using the Electronic portal imaging device (EPID) coupled with the treatment planning system (TPS), to calculate the doses delivered and verify agreement with the treatment plan. The results of QA analysis using the EPID, Delta ${ }^{4}$ and fluence calculations using the multi-leaf collimator (MLC) dynalog files on 10 IMRT patients are presented in this study.
\end{abstract}

Methods: EPID Fluence Images in integrated mode and Dynalog files for each field were acquired for 10 IMRT (6MV) patients and processed through an in house MatLab program to create an opening density matrix (ODM) which was used as the input fluence for dose calculation with the TPS (Pinnacle ${ }^{3}$, Philips). The EPID used in this study was the aSi1000 Varian on a Novalis TX linac equipped with high definition MLC. The resulting dose distributions were then exported to VeriSoft (PTW) where a 3D gamma was calculated using $3 \mathrm{~mm}-3 \%$ criteria. The Scandidos Delta ${ }^{4}$ phantom was also used to measure a $2 \mathrm{D}$ dose distribution for all 10 patients and a 2D gamma was calculated for each patient using the Delta ${ }^{4}$ software.

Results: The average 3D gamma for all 10 patients using the EPID images was $98.2 \% \pm 2.6 \%$. The average $3 \mathrm{D}$ gamma using the dynalog files was $94.6 \% \pm 4.9 \%$. The average $2 \mathrm{D}$ gamma from the Delta ${ }^{4}$ was $98.1 \% \pm 2.5 \%$. The minimum $3 \mathrm{D}$

Presenting author: Dewayne Lee Defoor; Department of Radiation Oncology, University of Texas Health Science Center at San Antonio, San Antonio, TX, USA.

Cite this article as:

Defoor D, Mavroidis P, Quino L, Gutierrez A, Papanikolaou N, Stathakis S. On the evaluation of patient specific IMRT QA using EPID, dynalog files and patient anatomy. Int J Cancer Ther Oncol 2014; 2(2):020219. DOI: 10.14319/ijcto.0202.19 gamma for the EPID and dynalog reconstructed dose distributions was found on the same patient which had a very large PTV, requiring the jaws to open to the maximum field size.

Conclusion: Use of the EPID, combined with a TPS is a viable method for QA of IMRT plans. A larger ODM size can be implemented to accommodate larger field sizes. An adaptation of this process to Volumetric Arc Therapy (VMAT) is currently under way.

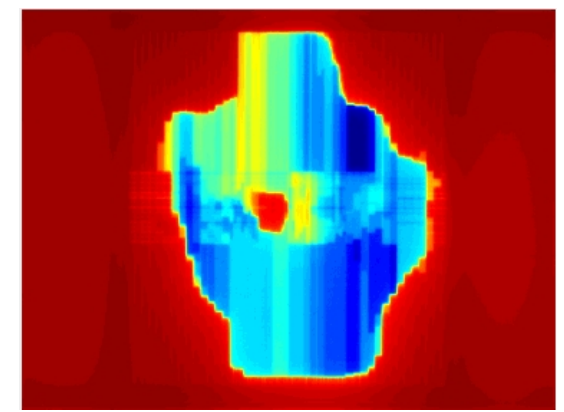

FIG 1: Fluence map measured by the EPID.

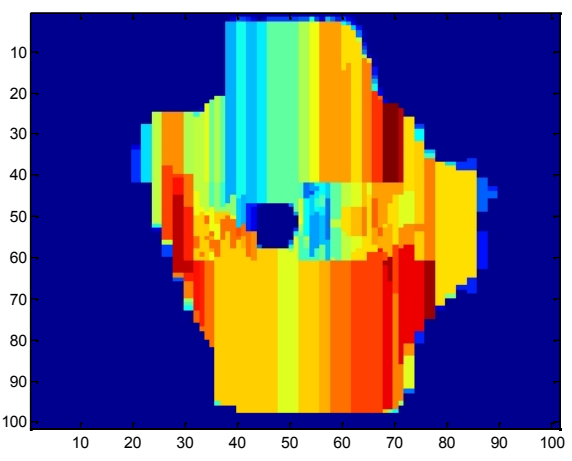

FIG 2: ODM created in Matlab from fluence map. 
TABLE 1: 3D Gammas at 3\% 3mm for all 10 patients per QA method.

\begin{tabular}{|c|c|c|c|c|c|c|c|c|c|c|c|}
\hline & 1 & 2 & 3 & 4 & 5 & 6 & 7 & 8 & 9 & 10 & \\
\hline & Liver & Liver & Liver & Liver & Liver & Lung & Lung & Lung & Lung & Pelvis & Average \\
\hline EPID & $100 \%$ & $98.1 \%$ & $99.9 \%$ & $97.6 \%$ & $98.7 \%$ & $100 \%$ & $98.6 \%$ & $99.1 \%$ & $98.8 \%$ & $91.4 \%$ & $98 \% \pm 2.6 \%$ \\
\hline Dynalog & $98.2 \%$ & $93.9 \%$ & $98.7 \%$ & $88.9 \%$ & $95.2 \%$ & $99.3 \%$ & $99.3 \%$ & $97.1 \%$ & $94.4 \%$ & $85.0 \%$ & $94.6 \% \pm 4.9 \%$ \\
\hline Delta $^{4 x}$ & $91.7 \%$ & $96.9 \%$ & $100 \%$ & $100 \%$ & $92.6 \%$ & $99.8 \%$ & $99.8 \%$ & $98.3 \%$ & $99.6 \%$ & $96.3 \%$ & $98.1 \% \pm 2.5 \%$ \\
\hline
\end{tabular}

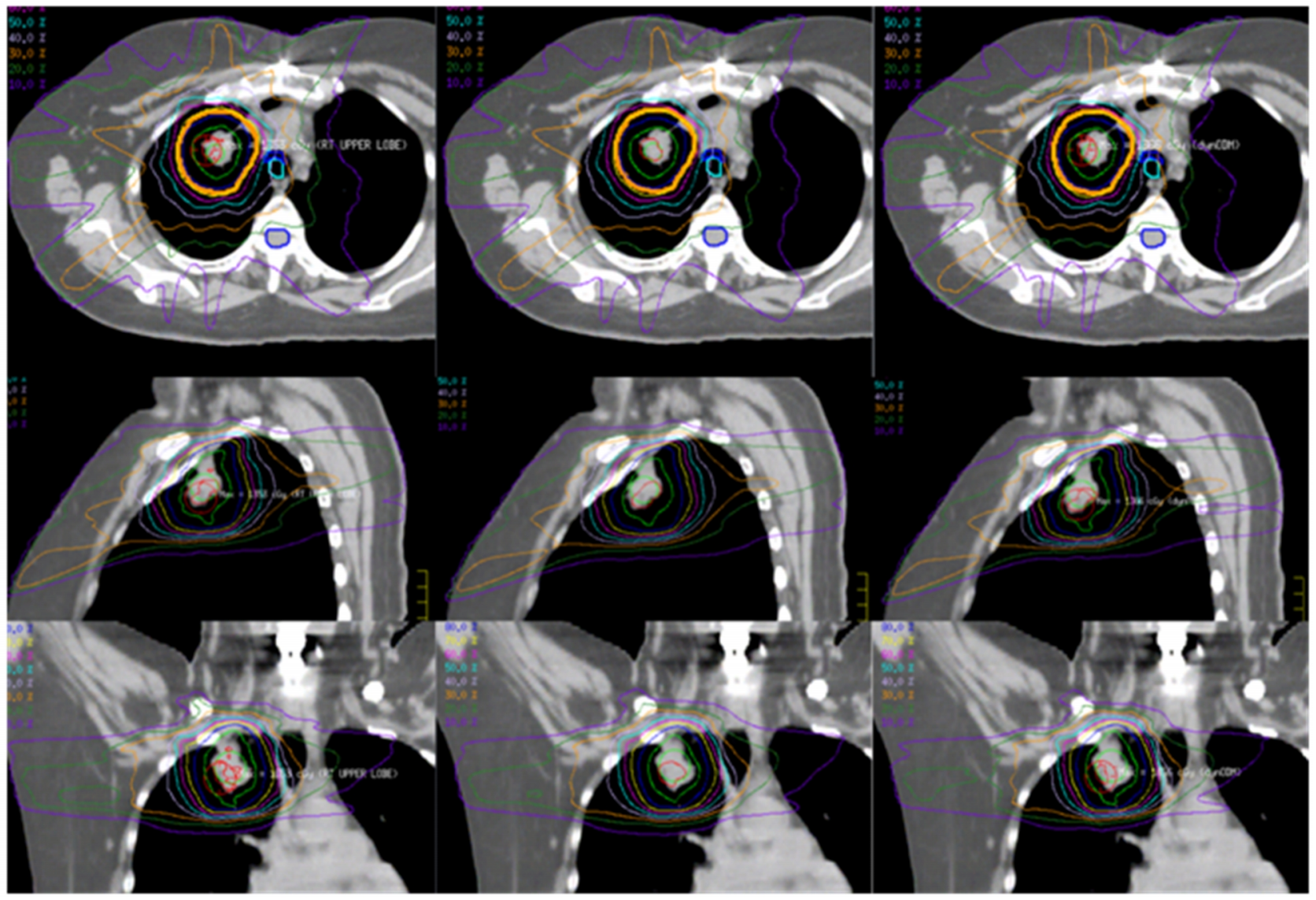

FIG. 3: Dose distributions calculated in Pinnacle for the approved plan (left) EPID ODM (middle) and dynalog ODM (right). 